\title{
Successful pregnancy outcome in two patients with pyonephrosis treated with percutaneous nephrostomy
}

\author{
Wansalan Karu Shullai', Manika Agarwal' ${ }^{2}$, Kalyani Reddy³, AS Singh ${ }^{4}$ \\ ${ }^{1}$ Senior Resident, Department of Obstetrics and Gynaecology, Lady Hardinge Medical College, New Delhi, ${ }^{2}$ Associate Professor, Department \\ of Obstetrics and Gynaecology, North Eastern Indira Gandhi Regional Institute of Health and Medical Sciences, Shillong, Meghalaya, \\ ${ }^{3}$ Senior Resident, Department of Obstetrics and Gynaecology, North Eastern Indira Gandhi Regional Institute of Health and Medical Sciences, \\ Shillong, Meghalaya, ${ }^{4}$ Professor and Head Professor, Department of Obstetrics and Gynecology, North Eastern Indira Gandhi Regional \\ Institute of Health and Medical Sciences, Shillong, Meghalaya.
}

A B S T R A C T

Urinary tract infections in pregnancy are common in the form of cystitis and pyelonephritis. Rarely patients may have pyonephrosis. Some of these patients may respond to antibiotic therapy. However those who do not, can be treated by drainage of pus under USG guided percutaneous nephrostomy. Though invasive procedures are generally avoided in pregnancy, however if required, it should be undertaken judiciously or the primary condition may aggravate which may lead to adverse pregnancy outcome. These cases are being presented to highlight that appropriate intervention in the form of percutaneous nephrostomy, is associated with favorable maternal and fetal outcome.

\section{Access this article online}

Website:

http://nepjol.info/index.php/AJMS DOI: 10.3126/ajms.v7i2.10446 E-ISSN: 2091-0576 P-ISSN: 2467-9100

Key words: Pregnancy, Percutaneous nephrostomy, Pyonephrosis

\section{INTRODUCTION}

Pyonephrosis comes from two Greek words pyon "pus" + nephros "kidney". Pyonephrosis can be defined as the presence of pus in an obstructed renal collecting system.

Pyonephrosis may present with a classic triad of fever, flank pain, and hydronephrosis, or simply hydronephrosis and sepsis. Due to the high risk of sepsis from pyonephrosis and the associated morbidity and mortality, early recognition and treatment of this condition is of paramount importance.

We present two cases in which pregnancy complicated by Pyonephrosis were successfully treated by USG guided percutaneous nephrostomy.

\section{CASE 1}

A 28 yr old $G_{3} A_{2} L_{0}$ patient was admitted at 30 wks with complaints of burning micturation, fever and pain in loin region. On examination there was right renal angle tenderness. Urinary culture examination was suggestive of significant E.coli infection, sensitive to nitrofurantoin and USG showed pyonephrosis of right kidney. Fetal evaluation on clinical examination and ultrasound was within normal limits. AFI was $8 \mathrm{~cm}$. Patient was started on nitrofurantoin and clinically improved. Patient was discharged with advice to continue nitrofurantoin for 3 wks. After 4 wks, patient's symptoms recurred and a USG guided right sided percutaneous nephrostomy as advised by urologist was done and pus was drained out. Pus was sterile on culture report.

Ten days later, when patient was at 34 wks 6 days a repeat scan showed oligohydramnios with amniotic fluid index less than $5 \mathrm{~cm}$. Patient was given a course of betamethasone for fetal lung maturity and $24 \mathrm{hrs}$ later lower segment caesarean section was done. An active female baby weighing $1.75 \mathrm{~kg}$ was delivered. Post caesarean both mother and baby did well. On the advice of urologist, nephrostomy tube was removed on post op day 5 , after remaining in situ for 21 days, as patient showed no evidence of urinary infection. Mother with baby were discharged on post op day 7 in healthy condition. Patient came for post natal check up after 6 weeks and her condition was satisfactory. 


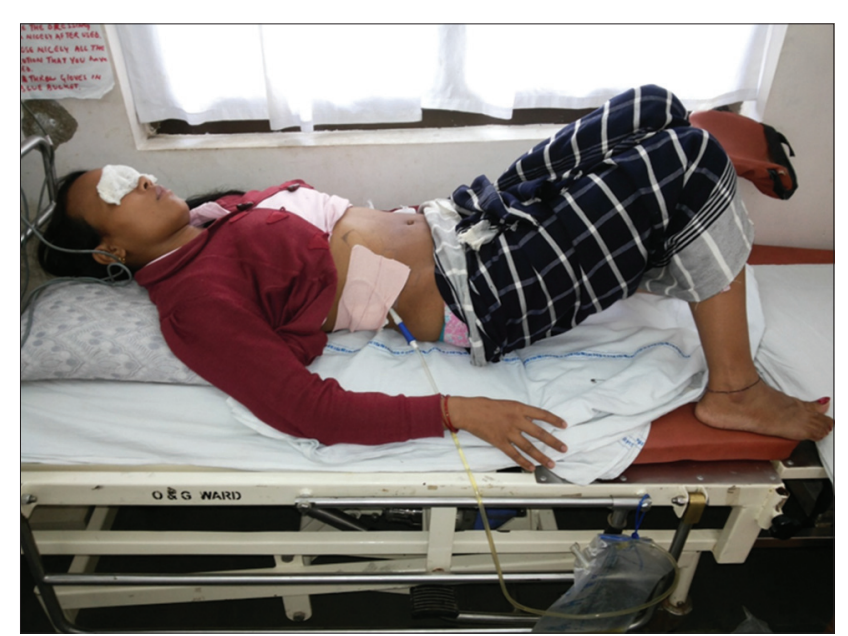

Figure 1: Case 1

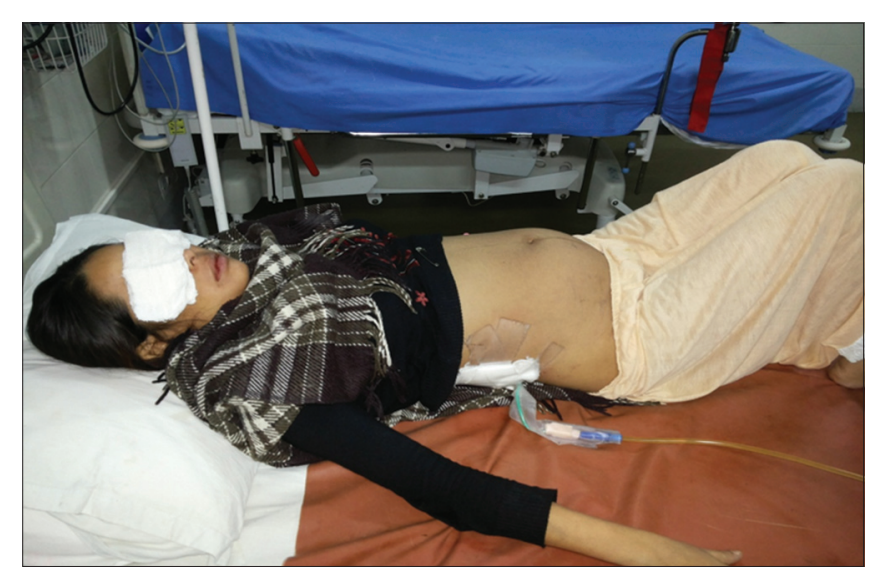

Figure 2: Case 2

Case 2

A 23 year old primi gravidae was admitted at 28 weeks of gestation with complaints of pain right lower abdomen. Examination revealed right ureteric colic and right hydronephrosis which was diagnosed on ultrasound. Fetal wellbeing on clinical examination and on ultrasound was within normal limits.

Patient did not respond to empirical therapy and as per the advice of the urologist a per cutaneous nephrostomy was done four days after admission. Patients symtoms were releaved and her condition on discharge was satisfactory.

Patient had regular antenatal visits and at 38 weeks she went into spontaneous labour. She had a normal vaginal delivery. A healthy male baby weighing $3 \mathrm{kgs}$ was delivered.

On advice of the urologist the PCN tube was removed eleven days post delivery after remaining in situ for 75 days. Mother and baby were discharged on post natal day eleven under satisfactory conditions. On post natal check up six weeks after delivery, the patient had no complaints.

\section{DISCUSSION}

Pregnancy can be complicated by true ureteral obstruction resulting from urolithiasis or mechanical ureteral compression secondary to uterine enlargement. Conservative measures result in clinical resolution in $70 \%-80 \%$ of women with ureteral obstruction during pregnancy. ${ }^{1}$ Although nephrostomy has been advocated for those in whom conservative therapy fails. ${ }^{1}$ Published data on its clinical value and safety are limited.

The maternal and fetal complications of pyonephrosis during pregnancy can be devastating, thus there is need for urgent and safe intervention. Emergency percutaneous nephrostomy is a potentially lifesaving procedure with a high technical success rate, minimal morbidity and long safety records that is often used in the setting of an obstructed and infected renal system i.e. pyonephrosis. ${ }^{2}$ Morbah A et al in their study of purulent retention in the upper urinary tract and pregnancy in 3 cases have suggested that when possible, nephrostomy constitutes an excellent means of drainage, while expecting a final disappearance of obstruction. ${ }^{3}$

Pyonephrosis if not adequately treated may have life threatening consequences. A case of peritonitis caused by spontaneous rupture of pyonephrosis in pregnancy has been reported. ${ }^{4}$ This patient required nephrectomy and abdominal drainage. The post-operative complication was septic shock requiring resuscitation and artificial ventilation and prolonged convalescence.

A case of laparoscopic nephrectomy for pyonephrosis during pregnancy has been reported. ${ }^{5}$ However, in a properly selected patient percutaneous nephrostomy would be a far safer and less invasive procedure for treatment of pyonephrosis in pregnancy.

\section{CONCLUSION}

Diagnosis of true urinary tract obstruction during pregnancy is very important as delay in diagnosis may lead to complications, one being pyonephrosis.

Pyonephrosis in pregnancy should be treated aggressively. If patient is not responding to antibiotics, performance of USG guided percutaneous nephrostomy is a life saving procedure. Untreated pyonephrosis may lead to complications as peritonitis, septic shock etc which may lead to an adverse maternal and fetal outcome.

\section{BIBLIOGRAPHY}

1. Biyani CS and Joyce AD. Urolithiasis in pregnancy I and II: pathophysiology, foetal considerations and diagnosis and management. BJU Int 2002; 89:811-823. 
2. Li AC and Regalado SP. Emergent percutaneous nephrostomy for the diagnosis and management of pyonephrosis. Semir Intervent Radiol 2012; 29(3):218-225.

3. Morbah A, Bibi M and Gueramazi HT. Purulent retention in the upper urinary tract and pregnancy. Apropos of 3 cases. Ann Urol (Paris) 1999;33(1):31-35.
4. Rabii R, Rais H, Sarf I, Joval A, Abontaieb R, Bennani S, et al. Peritonitis caused by spontaneous rupture of pyonephrosis in pregnancy report of a case. Ann Urol (Paris)1999;33(1):31-35.

5. Arvend NK, Singh O, Gupta SS, Sahay S and Dharaskar A. A Laparoscopic nephrectomy for pyonephrosis during pregnancy case report ans review of literature Rev Urol 2011; 13 (2): 98-103.

Authors Contribution:

WKS - Conceptualized study, literature search, statistically analyzed and interpreted, prepared first draft of manuscript and critical revision of the manuscript

MA - Concept, collected data and review of literature and helped in preparing first draft of manuscript; KR - Concept and design of the study, reviewed the literature, manuscript preparation and critical revision of the manuscript; SS - Concept of study, collected data and review of study.

Source of Support: Nil, Conflict of Interest: None. 